\title{
GREEN SYNTHESIS, CHARACTERIZATION, AND ANTIBACTERIAL ACTIVITY OF ZINC OXIDE NANOPARTICLE
}

\author{
C M NOORJAHAN* \\ Department of Zoology, Justice Basheer Ahmed Sayeed College for Women (Autonomous), Chennai, Tamil Nadu, India. \\ Email: cmnoorjahan@gmail.com
}

Received: 18 December 2018, Revised and Accepted: 11 February 2019

\section{ABSTRACT}

Objectives: Zinc oxide ( $\mathrm{ZnO}$ ) nanoparticles have received considerable attention due to their antimicrobial, UV blocking, and high catalytic and photochemical activities. Hence, an investigation has been carried out to synthesize the ZnO nanoparticle using aqueous Phyllanthus niruri (Keezhanelli) leaf extract. Aims and objectives of the present study are to synthesize using Keezhanelli (P. niruri) leaf extract, to study its characterization, and to determine its antibacterial activity.

Methods: Green synthesized ZnO nanoparticle was characterized by Fourier transform infrared (FTIR), scanning electron microscope (SEM), and transmission electron microscope (TEM) analysis. Antimicrobial activity of ZnO nanoparticle was carried out using agar well diffusion method.

Results: The result of the synthesized ZnO nanoparticle using Keezhanelli (P. niruri) leaf extract showed the change of color from pale white to brown color. The result of FTIR analysis of green synthesized $\mathrm{ZnO}$ nanoparticle revealed the presence of biomolecules such as polyphenols, flavonoids, alkaloids, polysaccharide, amino acid, and proteins. The result of the SEM studies showed that the green synthesized ZnO nanoparticle was spherical and cylindrical in shape. The size of the $\mathrm{ZnO}$ nanoparticle was recorded to be $5 \mu \mathrm{m}$. The result of TEM studies of $\mathrm{ZnO}$ nanoparticle showed that majority of the particles were spherical in shape with the size of $2 \mu \mathrm{m}$. The result of antibacterial activity against four bacterial species showed that green synthesized $\mathrm{ZnO}$ nanoparticle was found to be efficient in inhibiting the growth of the bacterial isolates. Maximum zone formation was exhibited against Staphylococcus saprophyticus.

Conclusion: Thus, from the results of the present study, it can be concluded that synthesis of $\mathrm{ZnO}$ nanoparticle using leaf extract of Keezhanelli (P. niruri) has several advantages such as simple, cost-effective, time consuming, safe, and eco-friendly compared to other methods of nanoparticle synthesis as evidenced in the present study.

Keywords: Phyllanthus niruri (Keezhanelli) leaf extract, Zinc oxide nanoparticle, Fourier transform infrared, Scanning electron microscope, Transmission electron microscope, Antibacterial activity.

(c) 2019 The Authors. Published by Innovare Academic Sciences Pvt Ltd. This is an open access article under the CC BY license (http://creativecommons. org/licenses/by/4. 0/) DOI: http://dx.doi.org/10.22159/ajpcr.2019.v12i4.30961

\section{INTRODUCTION}

Nanotechnology is the production and use of materials at the smallest possible scale [1]. In nanotechnology, a nanoparticle is defined as a small object that behaves as a whole unit in terms of its transport and properties [2]. In the current situation, one of the most promising and therapeutic agents is nanoparticles. Nanoparticles are being viewed as the fundamental building blocks of nanotechnology [3].

In general, metal oxide nanoparticles are inorganic. Various nanoparticles such as $\mathrm{Fe}, \mathrm{Ni}, \mathrm{Co}, \mathrm{Mn}$, and $\mathrm{Zn}$ are known as the enormously accepted magnetic materials for a wide range of applications such as various electronic ignition systems, generators, vending machines, medical implants, wrist watches, inductor core, transformer circuits, magnetic sensors and recording equipment, telecommunications, magnetic fluids, and microwave absorbers [4]. Metal-based nanoparticles constitute an effective antimicrobial agent against common pathogenic microorganisms. A multiple of research have established that zinc oxide $(\mathrm{ZnO})$ nanoparticle has antifungal and antibacterial activity.

$\mathrm{ZnO}$ is an inorganic compound with the formula $\mathrm{ZnO}$. It usually appears as a white powder, nearly insoluble in water. The powder is widely used as an additive in numerous materials and products including plastics, ceramics, glass, cement, rubber (e.g., car tyres), lubricants, paints, ointments, adhesives, pigments, foods (source of $\mathrm{Zn}$ nutrient), batteries, ferrites, and fire retardants. $\mathrm{ZnO}$ is present in the earth crust as a mineral zincite; however, most $\mathrm{ZnO}$ used commercially is produced synthetically [5].

Green synthesis of nanoparticles has gained significant importance in recent years and has become one of the most preferred methods of the synthesis of nanoparticle. Green synthesis of nanoparticle aims to protect the environment not only by cleaning up but also by inventing new chemical process that does not defile [6]. The uses of plant extracts are far more advantageous than that of other biological methods of synthesis since this process eliminates the tedious task of maintaining microbial medium and cultures [5]. Based on literature survey, $\mathrm{ZnO}$ nanoparticle was synthesized using Phyllanthus niruri (Keezhanelli) leaves which is distributed throughout the tropical and subtropical regions of both hemispheres. Thus, the aims and objectives of the present study are to green synthesize $\mathrm{ZnO}$ nanoparticle using Keezhanelli (P. niruri) leaf extract, to characterize green synthesized $\mathrm{ZnO}$ nanoparticle using Fourier transform infrared (FTIR), scanning electron microscope (SEM), and transmission electron microscope (TEM) analysis and also to determine the antibacterial activity of green synthesized $\mathrm{ZnO}$ nanoparticle using agar well diffusion method.

\section{METHODS}

Preparation of zinc acetate dihydrate solution for the synthesis of ZnO nanoparticle

Zinc acetate dihydrate with $90 \%$ purity was obtained from Hi-Media and distilled water was used throughout the experiments for the synthesis of $\mathrm{ZnO}$ nanoparticle. 


\section{Selection of plant}

Fresh and healthy Keezhanelli ( $P$. niruri) leaves were collected from the tree. The leaves were ensured that they were healthy, uninfected, thoroughly washed, and rinsed with sterile distilled water.

\section{Authentication of plant material}

The fresh leaves of Keezhanelli ( $P$. niruri) collected and it was identified and authenticated by Prof. P. Jayaraman, Taxonomist, Plant Anatomy Research Centre (PARC), Chennai (Certificate No. PARC/2018/3882).

\section{Collection of bacterial isolates for antibacterial activity of $\mathrm{ZnO}$} nanoparticle

Clinical isolates such as Bacillus sp., Staphylococcus epidermidis, Staphylococcus saprophyticus, and Escherichia coli were collected from a tertiary hospital. Samples were transported to the laboratory for further processing in an ice box.

Green synthesis of ZnO nanoparticle using Keezhanelli leaf extract Preparation of zinc acetate dihydrate solution for the synthesis of $\mathrm{ZnO}$ nanoparticle was carried out by following the procedure of Senthilkumar et al. [7]. 0.2 M of zinc acetate dihydrate was dissolved in $70 \mathrm{ml}$ of distilled water and stirred for few minutes. Preparation of leaf extract from Keezhanelli ( $P$. niruri) plant was carried out by following the procedure of Kanthimathi and Soranam [8]. $10 \mathrm{~g}$ of the fresh green leaves of Keezhanelli (P. niruri) was added to $100 \mathrm{ml}$ of distilled water and magnetically stirred for $2 \mathrm{~h}$ at $80^{\circ} \mathrm{C}$. After cooling to room temperature and filtering through Whatman No. 1 paper, $30 \mathrm{ml}$ of this Keezhanelli leaf extract was mixed homogeneously with the already prepared zinc acetate solution. The reacted solution was dried at $60^{\circ} \mathrm{C}$ overnight to yield pale white color $\mathrm{ZnO}$ nanoparticle, which was finally calcined at $100^{\circ} \mathrm{C}$ for $1 \mathrm{~h}$ and preserved in airtight vials for further studies.

\section{Characterization of $\mathrm{ZnO}$ nanoparticle}

\section{FTIR spectroscopy}

The FTIR spectra of $\mathrm{ZnO}$ nanoparticle of $P$. niruri powder were recorded in SHIMADZU-8400 spectrometer using $\mathrm{KBr}$ pellet method.

\section{SEM}

In the present work, SEM machine was employed to study the morphology of green synthesized nanoparticle. The experiment was performed at an accelerating voltage of $20 \mathrm{kV}$. The slide was coated with platinum, and after the platinum coating, the SEM image was taken.

\section{TEM}

TEM analysis of green synthesized $\mathrm{ZnO}$ nanoparticle was done using Philips (Technai 10). Thin films of sample were prepared on a carboncoated copper grid by just dropping a very small amount of sample on the grid, extra solution was removed using a blotting paper, and then, the film on the TEM grid was allowed to dry by putting it under incubator. The images were obtained by Technai, Twin $200 \mathrm{KV}$, and a bias voltage of $200 \mathrm{kV}$ was used to analyze sample.

\footnotetext{
Antibacterial activity of green synthesized $\mathrm{ZnO}$ nanoparticle using agar well diffusion method

The effect of Keezhanelli (P. niruri) leaves extract on the test organisms was assayed by agar well diffusion method. Mueller-Hinton agar medium was poured into the Petri plates aseptically and was allowed to solidify. The lawns of the test bacterial strains were done with the help of sterile cotton swab. Wells were made with the help of sterile cork borer ( $6 \mathrm{~mm})$, and the cut agar discs were removed aseptically with sterile forceps. $100 \mu \mathrm{l}$ of green synthesized $\mathrm{ZnO}$ nanoparticle was added into the wells. The test plates were incubated aerobically at $37^{\circ} \mathrm{C}$ for $24 \mathrm{~h}$. After incubation, the results were recorded based on the presence or absence of inhibition zone. The antibacterial activity of green synthesized $\mathrm{ZnO}$ nanoparticle was assayed by measuring the diameter of the inhibition zone formed around the wells.
}

Statistical analysis

The data obtained from the experiments were analyzed and expressed as mean and standard deviation.

$$
\mathrm{S} . \mathrm{D}=\sqrt{\frac{1}{N-1} \sum x^{2}-\frac{\left(\sum x^{2}\right)}{N}}
$$

Where, $N=$ Number of individual observation. $\sum x^{2}=$ Sum of square of individual observation. $\left(\sum x^{2}\right)=$ Square of the total individual observation.

$$
\text { Chi - square test }=\chi^{2}=\Sigma \frac{(O-E)^{2}}{E}
$$

Where, $\mathrm{O}=$ observed values

$\mathrm{E}=$ expected values.

\section{RESULTS}

Green synthesis of ZnO nanoparticle using Keezhanelli (P. niruri) leaf extract

The results of the green synthesis of $\mathrm{ZnO}$ nanoparticle using Keezhanelli ( $P$. niruri) leaves extract are shown in plate 1 . The results of the study revealed that the color of the sample changed from pale white to brown color which was due to the reduction of zinc ions into zinc nanoparticle that occurred due to the surface plasmon resonance phenomenon. The metal nanoparticle has free electrons, which helps in the formation of the surface plasmon resonance absorption band.

\section{characterization of green synthesized ZnO nanoparticle}

Further studies were extended to characterize the green synthesized nanoparticle using FTIR spectroscopy, SEM, and TEM analysis.

The results of FTIR absorption spectra of green synthesized ZnO nanoparticle are presented in Fig. 1. The FTIR spectroscopy analysis of $\mathrm{ZnO}$ nanoparticle has revealed the presence of different peaks pertaining to various functional groups.

The results of FTIR study showed that the peaks at $3104 \mathrm{~cm}^{-1}-3433 \mathrm{~cm}^{-1}$ were due to stretching vibration of $\mathrm{N}-\mathrm{H}$ groups in amines. $\mathrm{C}=0$ stretching in polyphenols and carboxyl was observed at $1498-1565 \mathrm{~cm}^{-1}$, bands at $1190-1421 \mathrm{~cm}^{-1}$ were attributed to the $\mathrm{C}=\mathrm{C}$ stretching in aromatic ring. $\mathrm{C}-0$ stretching in amino acids showed a band at $923-932 \mathrm{~cm}^{-1}$. Bands at $632-680 \mathrm{~cm}^{-1}$ were due to $\mathrm{C}-\mathrm{H}$ binding in aliphatic region. In addition to the absorption bands of these biomolecules, two new peaks appear at $615-618 \mathrm{~cm}^{-1}, 505-510 \mathrm{~cm}^{-1}$, and $490-494 \mathrm{~cm}^{-1}$ in the IR spectrum of the $\mathrm{ZnO}$ nanoparticles which are the characteristic peaks of $\mathrm{ZnO}$ nanoparticle.

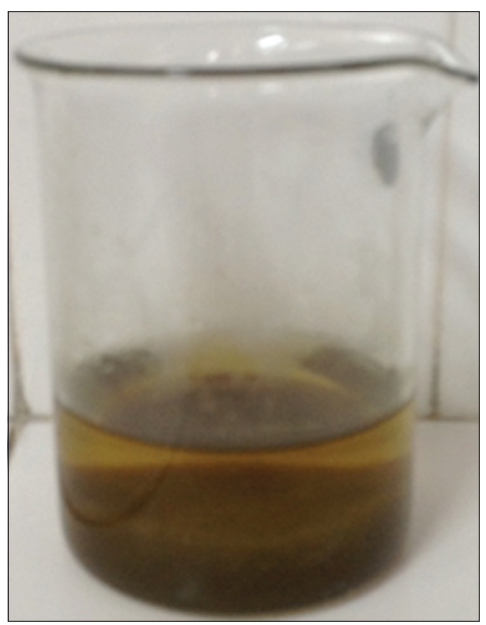

Plate 1: Green synthesis of zinc oxide nanoparticle using Keezhanelli (Phyllanthus niruri) leaves extract. 


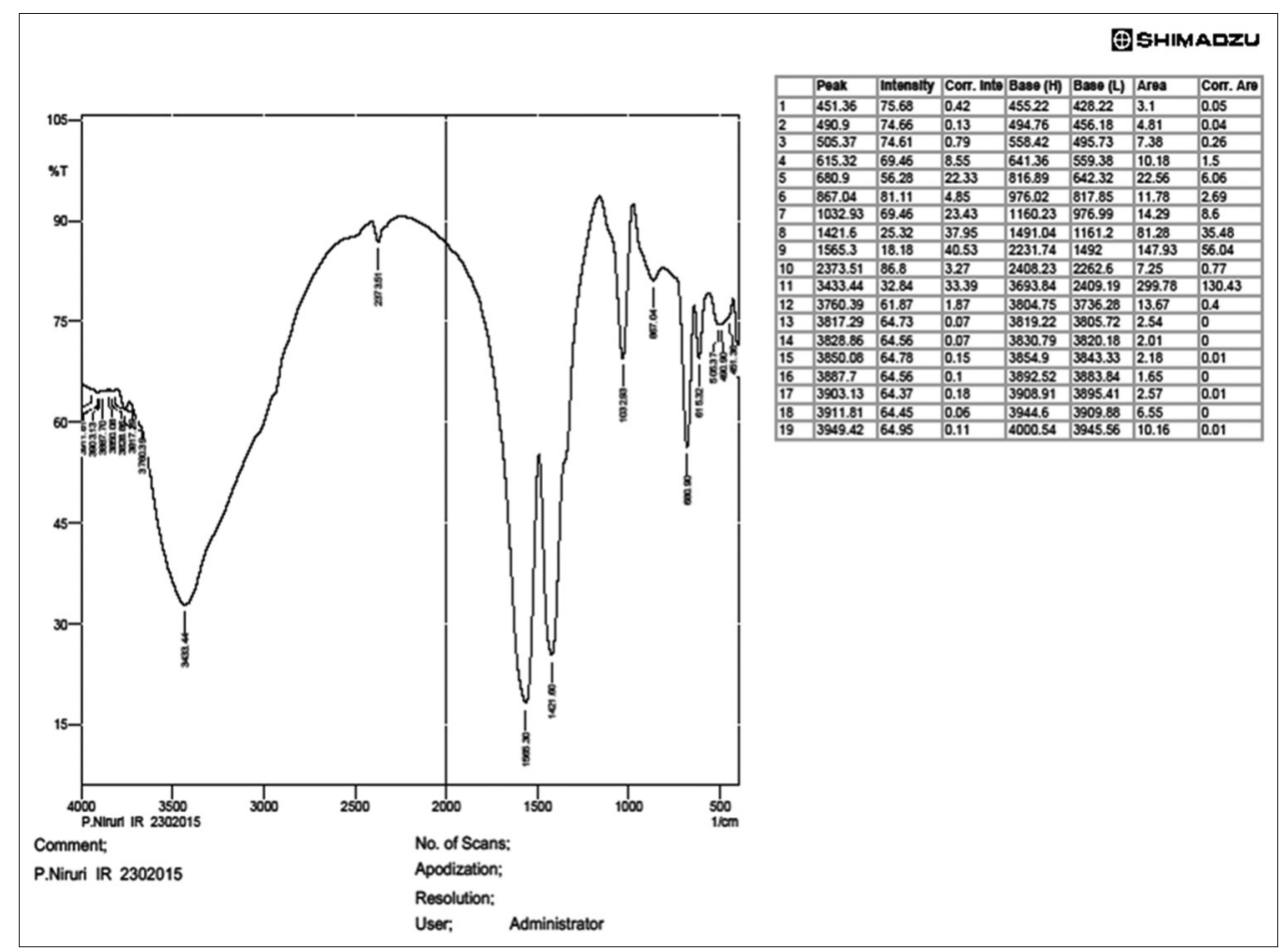

Fig. 1: Fourier transform infrared analysis of green synthesized zinc oxide nanoparticle using leaves extract of Keezhanelli (Phyllanthus niruri)

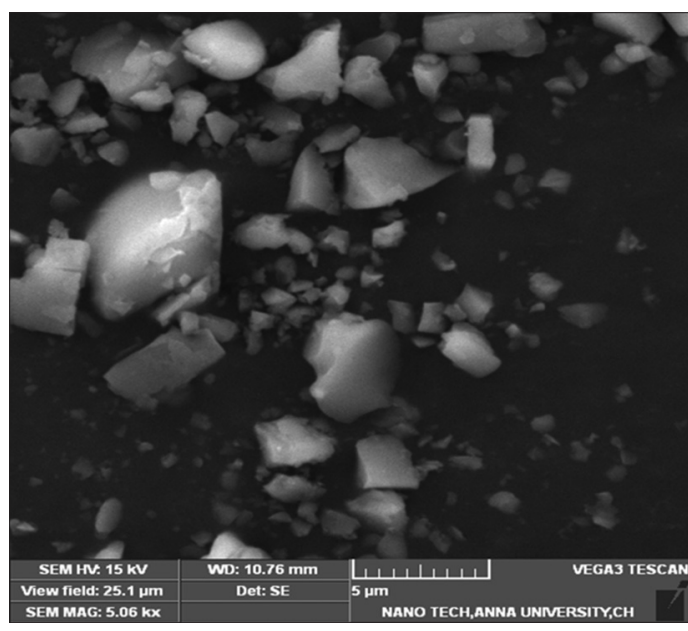

Plate 2: Scanning electron microscope analysis of green synthesized zinc oxide nanoparticle using leaves extract of Keezhanelli (Phyllanthus niruri)

From the results of FTIR spectrum, it can be observed that $\mathrm{ZnO}$ nanoparticle is rich in polyphenols, alkaloids, flavonoids, etc. The involvement of these biomolecules in the reduction and stabilization (capping actions) is clearly evident from the IR spectrum of the green synthesized $\mathrm{ZnO}$ nanoparticle.

The results of the SEM studies on $\mathrm{ZnO}$ nanoparticles using Keezhanelli (P. niruri) leaves extractare depicted in Plate 2. Spherical-and cylindricalshaped nanocrystals were formed by exposing the leaf extracts of aforesaid plant to the aqueous zinc acetate dihydrate solution. The size of the $\mathrm{ZnO}$ nanoparticles synthesized using Keezhanelli (P. niruri) leaf extracts ranges from $5 \mu \mathrm{m}$ in diameter.

The results of the TEM analysis of green synthesized $\mathrm{ZnO}$ nanoparticle are depicted in Plate 3. TEM micrographs provide a clear idea on the

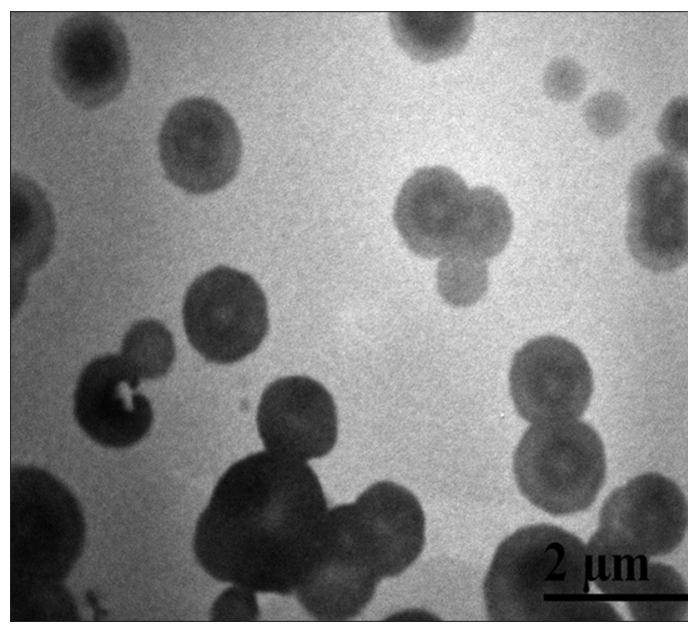

Plate 3: Transmission electron microscope analysis of green synthesized zinc oxide nanoparticle using leaves extract of Keezhanelli (Phyllanthus niruri)

shape of nanoparticle. Majority of the particles were spherical in shape with the size of $2 \mu \mathrm{m}$. These nanoparticles were well distributed without much aggregation.

\section{Antibacterial activity of green synthesized $\mathrm{ZnO}$ nanoparticle}

The results of the antibacterial activity of green synthesized $\mathrm{ZnO}$ nanoparticle against the bacterial isolates are shown in Table 1 . The result of the study showed that $\mathrm{ZnO}$ nanoparticle formed a zone whose size ranged from $16 \pm 0.8 \mathrm{~mm}$ to $29.6 \pm 1.2 \mathrm{~mm}$ in diameter. The maximum zone of $29.6 \pm 1.2 \mathrm{~mm}$ was exhibited toward S. saprophyticus and the least zone of $16 \pm 0.8 \mathrm{~mm}$ in diameter was shown by $S$. epidermidis. The values were significant at $0.1 \%$ level.

The result of the antibacterial activity of green synthesized nanoparticle against four bacterial species showed that green synthesized $\mathrm{ZnO}$ 
Table 1: Statistical data of agar well diffusion method of green synthesized zinc oxide nanoparticle

\begin{tabular}{lll}
\hline S. No. & Name of the organism & Mean \pm standard deviation \\
\hline 1. & Bacillus sp. & $16.3 \pm 1.2$ \\
2. & Escherichia coli & $18 \pm 0.81$ \\
3. & Staphylococcus epidermidis & $16 \pm 0.8$ \\
4. & Staphylococcus saprophyticus & $29.6 \pm 1.2$ \\
\hline
\end{tabular}

\pm : Standard deviation, The values were significant at $0.1 \%$ level

nanoparticle was found to be efficient in inhibiting the growth of the bacterial isolates.

\section{Statistical analysis}

The results of the statistical analysis of the data obtained from the above experiments revealed that the probability was found to be significant at $0.1 \%$ level.

\section{DISCUSSION}

Nanotechnology is a multidisciplinary scientific field undergoing explosive development. Nanometer-sized particles offer novel structural, optical, and electronic properties that are not attainable with individual molecules or bulk solids [9]. The characters of metal and metal oxide nanoparticles have been of great interest due to their distinctive features such as catalytic activity, optical, magnetic, and electrical properties [10].

Different types of physical and chemical methods are employed for the synthesis of nanoparticles. The use of these synthetic methods requires both strong and weak chemical reducing agents and protective agents such as sodium borohydride, sodium citrate, and alcohols. These agents are mostly toxic, flammable, cannot be easily disposed off due to environmental issues and also show a low production rate [11-14]. It leads to in search of alternatives which could be ecofriendly and does not cause any harm to live organisms including human. One such method is the biological method using microbes and plants either as reducing agents or protective agents. Many biological organisms, both unicellular and multicellular, are known to produce inorganic materials either intra- or extra-cellular, often of nanoscale dimensions and exquisite morphology and hierarchical assembly. The biosynthesis of nanoparticles employs the use of biological agents such as bacteria, fungi, actinomycetes, yeast, algae, and plants $[11,15]$. The rate of the reduction of metal ions using biological agents is found to be much faster and also at ambient temperature and pressure conditions. Hence, the present investigation was aimed to synthesize $\mathrm{ZnO}$ nanoparticle using $P$. niruri leaf extract, to characterize the biosynthesized $\mathrm{ZnO}$ nanoparticle, and to evaluate their antibacterial efficiency against some pathogenic bacterial isolates such as Bacillus sp., E. coli, S. epidermidis, and S. saprophyticus.

The results of the biosynthesis of $\mathrm{ZnO}$ Nanoparticle using Keezhanelli $(P$. niruri) leaf extract showed the change of colour from pale white to brown colour which may be due to surface Plasmon Resonance phenomenon as ZnO Nanoparticle have free electrons which helps in the formation of the Surface Plasmon Resonance absorption band, that occurred due to the united vibration of the electrons of metal nanoparticles in resonance with light wave, which is supported by the work [7]

Further studies were extended to characterize the biosynthesized nanoparticle using FTIR spectroscopy, SEM, and TEM analysis.

The results of FTIR analysis of the biosynthesized nanoparticle revealed the presence of biomolecules such as alkaloids, flavonoids, and phenolic groups of molecules present in P. niruri leaf extract that is responsible for the reduction and amino acids and amide linkages in proteins were responsible for the stabilization process of the biosynthesized nanoparticle which was identified by FTIR analysis. The involvement of these biomolecules in the reduction and stabilization (capping actions) is clearly evident from the IR spectrum of the biosynthesized $\mathrm{ZnO}$ nanoparticle. The result of the above study was supported by the work [16].

SEM was used to determine size, location, and shape of the $\mathrm{ZnO}$ nanoparticle. The results of the SEM studies of biosynthesized nanoparticle showed that spherical- and cylindrical-shaped $\mathrm{ZnO}$ nanocrystals were formed by exposing the leaf extract of aforesaid plant to the aqueous zinc acetate dihydrate solution. The size of the $\mathrm{ZnO}$ nanoparticle synthesized using Keezhanelli (P. niruri) leaf extracts ranged from $69.71 \mathrm{~nm}$ to $94.36 \mathrm{~nm}$ in diameter [16].

TEM micrographs provide a clear idea on the shape of nanoparticles. Shape of ZnO nanoparticles of Keezhanelli leaf extract was spherical with the size of $2 \mu \mathrm{m}$. This nanoparticle was well distributed without any aggregations. The separation between the $\mathrm{ZnO}$ nanoparticles without any aggregation observed in the TEM image could be due to capping by proteins [16].

The result of the antibacterial activity of biosynthesized nanoparticles using Keezhanelli (P. niruri) leaf extract against four bacterial species showed that though biosynthesized $\mathrm{ZnO}$ nanoparticle was found to be efficient in inhibiting the growth of the bacterial isolates which may be due to the presence of different functional groups, proteins, amides, etc., present in biosynthesized ZnO nanoparticle using P. niruri leaf extract. This may be due to the electrostatic interaction between bacterial cell surface and nanoparticle for inhibiting the growth and also production of hydrogen peroxide from $\mathrm{ZnO}$ nanoparticle that leads to the entry of particle into bacterial cell membrane cause injury and finally causes the death of the bacterium [17]. Thus, the use of plant extract with known antimicrobial properties can be of great significant for therapeutic treatment. This is supported by the work [18].

Thus, the results of the above study show that the biosynthesized ZnO nanoparticle can be used in the treatment of wastewater and also in the development of antibacterial water filters for water purification as bacterial diseases are transmitted through water [19].

\section{CONCLUSION}

Thus, from the results of the above study, it can be concluded that green synthesis of $\mathrm{ZnO}$ nanoparticle using plants has several merits such as simple, inexpensive, good stability of nanoparticles, less time consumption, non-toxic byproducts, ecofriendly, and large-scale synthesis.

\section{AUTHORS' CONTRIBUTIONS}

All the practical and theoretical works were carried out by the author Dr. C.M. Noorjahan herself.

\section{CONFLICTS OF INTEREST}

The author declares that there are no conflicts of interest.

\section{REFERENCES}

1. Meruvu H, Vangalapati M, Chippada SC, Bammidi SR. Synthesis and characterization of zinc oxide nanoparticles and its antimicrobial activity against Bacillus subtilis and Escherichia coli. Rasayan J Chem 2011; $4: 217-22$ 
2. Prathna TC, Ashok MC, Mukherjee RA. Biomimetic synthesis of nanoparticles. Sci Technol Appl 2012;20:76-80.

3. Murugesan S, Bhuvaneswari S, Sivamurugan V. Green synthesis, characterization of silver nanoparticles of a marine red alga Spyridia fusiforms and their antibacterial activity. Int J Pharm Pharm Sci 2017;9:192-7.

4. Willard MA, Kurihara LK, Carpenter EE, Calvin S, Harris VG. Chemically prepared magnetic nanoparticles. Int Mater Rev 2014;49: 125-170.

5. Banerjee S. Green Synthesis and Characterization of Metal Nanoparticles and its Antimicrobial Properties. Ph.D. Thesis. Jadavpur: Jadavpur University; 2012. p. 9-10.

6. Pattanayak U. Green Synthesis of Zinc Nanoparticle by Microbes, Ph.D. Thesis, Department of Life Science. Rourkela: National Institute of Technology; 2013. p. 5.

7. Senthilkumar SR, Sivakumar T. Green Tea (Camellia sinensis) mediated synthesis of zinc oxide $(\mathrm{ZnO})$ nanoparticles and studies on their antimicrobial activities. Int J Pharm Pharm Sci 2014;6:55-60.

8. Kanthimathi M, Soranam R. Antibacterial effects of Emblica officinalis and Phyllanthus niruri crude extracts against bacterial pathogens. Int $\mathrm{J}$ Pharm Clin Sci 2013;3:20-3.

9. Sajja HK, East MP, Mao H, Wang YA, Nie S, Yang L. Development of multi-functional nanoparticles for targeted drug delivery and noninvasive imaging of therapeutic effect. Curr Drug Discov Technol 2009; 6:43-51.

10. Singhal G, Bhavesh R, Kasariya K, Sharma AR, Singh RP. Biosynthesis of silver nanoparticles using Octimum sanctum (Tulsi) leaf extract and screening its antimicrobial activity. J Nanopart Res 2011;13:2981-8.
11. Rai M, Yadav A, Gade A. Current trends in photosynthesis of metal nanoparticles. Crit Rev Biotechnol 2008;28:277-84.

12. Sharma NC, Sahi SV, Nath S, Parsons JG, Gardea-Torresdey JL, Pal T. Synthesis of plant-mediated gold nanoparticles and catalytic role of biomatrix-embedded nanomaterials. Environ Sci Technol 2007;41: 5137-42.

13. Bar H, Bhui DK, Sahoo GP, Sarkar P, De SP, Mirsa A. Green synthesis of silver nanoparticles using latex of Jatropha curcas. Colloids surf A: Physicochem Eng Asp 2009a;339:134-9.

14. Bar H, Bhui DK, Sahoo GP, Sarkar P, Pyne S, Mirsa A. Green synthesis of silver nanoparticles using seed extract of Jatropha curcas. Colloids surf A: Physicochem Eng Asp 2009b;348:212-6.

15. Thakkar KN, Mhatre SS, Parikh RY. Biological synthesis of metallic nanoparticles. Nanomedicine 2010;6:257-62.

16. Gnanasangeetha D, Sarala D, Thambavani. Facile and eco-friendly method for the synthesis of zinc oxide nanoparticles using Azadirachta and Emblica. Int J Pharm Sci Res 2014;5:2866-73.

17. Li X, Xing Y, Ding Y, Li W. Antimicrobial activities of ZnO powdercoated PVC film to inactivate food pathogens. Int J Food Sci Technol 2009;44:2161-8.

18. Shanmugam B, Shanmugam KR, Ravi S, Subbaiah GV, Mallikarjuna K, Reddy KS. Antibacterial activity and phytochemical screening of Phyllanthus niruri in ethanolic, methanolic and aqueous extracts. Int J Pharm Sci Rev Res 2014;27:85-9.

19. Shailesh R, Mustopa NW, Suryakanth RM, Kalilas DM, Sonawane KD. Ecofriendly production of silver nanoparticles using Candida utilis and mechanistic action against pathogenic microorganisms. 3 Biotech 2015; $5: 33-8$. 